This item was submitted to Loughborough's Research Repository by the author.

Items in Figshare are protected by copyright, with all rights reserved, unless otherwise indicated.

\title{
The new theory of electron transfer: application to the photosynthetic
} reaction centre

PLEASE CITE THE PUBLISHED VERSION

PUBLISHER

(C) Springer Berlin / Heidelberg

LICENCE

CC BY-NC-ND 4.0

REPOSITORY RECORD

Fletcher, Stephen. 2019. "The New Theory of Electron Transfer: Application to the Photosynthetic Reaction Centre". figshare. https://hdl.handle.net/2134/3458. 
This item was submitted to Loughborough's Institutional Repository by the author and is made available under the following Creative Commons Licence conditions.

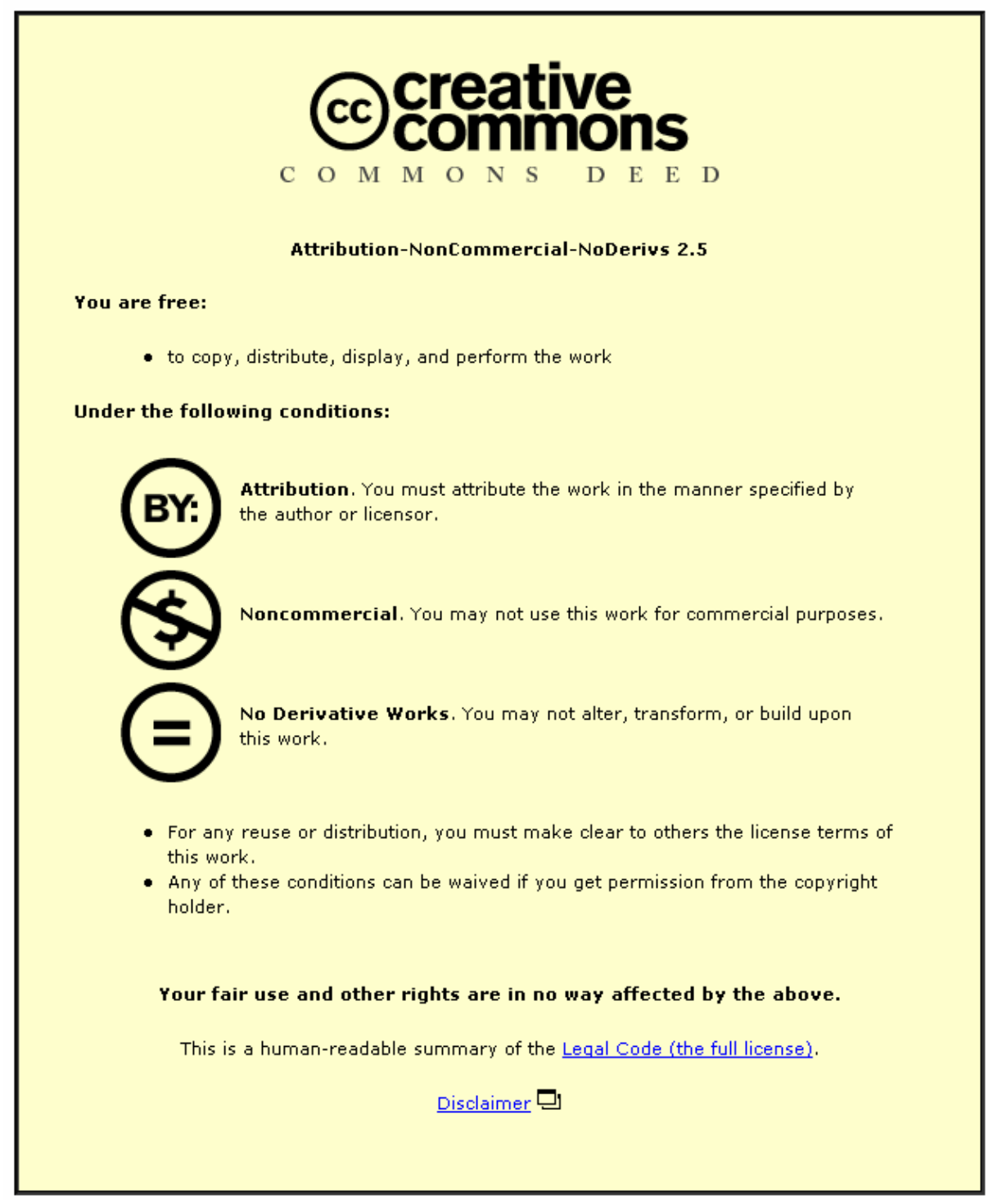

For the full text of this licence, please go to: http://creativecommons.org/licenses/by-nc-nd/2.5/ 
Ms. No. JSEL-D-08-00152R1

S Fletcher, Journal of Solid State Electrochemistry, 12, 1511-1520 (2008).

Submitted 16 May 2008 Accepted 02 Jun 2008 Published Online 27 Jun 2008

Published online first at http://dx.doi.org/10.1007/s10008-008-0609-0

The institutional repository is http://hdl.handle.net/2134/3458

The original publication at http://www.springerlink.com/content/h83417t843282096/

\title{
The New Theory of Electron Transfer. Application to the Photosynthetic Reaction Centre
}

\author{
Stephen Fletcher \\ Department of Chemistry, \\ Loughborough University, \\ Ashby Road, Loughborough, \\ Leicestershire LE11 3TU, UK
}

Tel. 01509222561 Fax 01509223925

Email Stephen.Fletcher@Lboro.ac.uk

\section{Keywords}

Electron Transfer, Photosynthesis, Equivalent Circuit, Gibbs Energy Profile, Inverted Region, Electron Transport Chain, Marcus Theory, Oxidative Stress. 


\begin{abstract}
Based on recent developments in the theory of electron transfer, we prove that a nonpolar environment is needed to maintain the high efficiency and chemical integrity of the photosynthetic reaction centre. We also determine the Gibbs energy diagram for the primary act of charge separation in photosynthesis, and propose an equivalent circuit that captures the principal features of the entire acceptor side of the electron transport chain in photosystem II.
\end{abstract}

\title{
Introduction
}

In two previous papers a new (non-Marcus) theory of electron transfer was developed, and the results were applied to a model system over a wide range of thermodynamic driving forces $[1,2]$. In this paper, we apply the results to a real system, namely the photosynthetic reaction centre.

The new theory takes into account the fact that charge fluctuations contribute to the activation of electron transfer, besides dielectric fluctuations. When charge fluctuations are included, the results turn out to be radically different from those of previous theories, particularly those of Marcus [3-9] and Hush [10-13]. Most importantly, it is found that highly polar environments (i.e. environments having high relative permittivity) are able to catalyze the rates of thermally-activated electron transfer processes, because, under certain well-defined conditions, they are able to stabilize the transient charges that develop on transition states. This important effect is absent from Marcus-Hush theories, but is well described by the Fletcher theory $[1,2]$. Some consequences of its inclusion can be seen in plots of rate constant for electron transfer versus driving force, as shown in Fig.1. On the new theory, the relative permittivity of the environment exerts a powerful influence on the reaction rate in the highly exergonic region (the "inverted" region) and in the highly endergonic region (the "superverted" region).

Fig. 1 is drawn on the assumption that electron transfer is non-adiabatic and proceeds according to Dirac's time-dependent perturbation theory [14]. Experimentally, we expect non-adiabatic electron transfer to be observed whenever there is small orbital overlap (weak coupling) between donor and acceptor states, so that overall electron transfer rates are "slow" (ten picosecond timescale, or longer, at room temperature). This encompasses most cases of biological interest.

Dirac's theory applies to any system that is undergoing a transition from one electronic state to another, in which the energies of the states are briefly equalized by fluctuations in the environment. If we assume that the relative probability of observing a fluctuation from state $i$ to state $j$ at temperature $T$ is given by the Boltzmann factor $\Delta G_{\mathrm{ij}} / k_{\mathrm{B}} T$, then one finds

$k_{\mathrm{et}}=\frac{2 \pi}{\hbar} H_{\mathrm{DA}}^{2} \frac{1}{\sqrt{4 \pi \lambda k_{\mathrm{B}} T}} \exp \left(\frac{-\left(\lambda+\Delta G^{0}\right)^{2}}{4 \lambda k_{\mathrm{B}} T}\right)$

where $k_{\mathrm{et}}$ is the rate constant for electron transfer, $H_{\mathrm{DA}}$ is the electronic coupling matrix element between the electron donor and acceptor species, $k_{\mathrm{B}}$ is the Boltzmann 
constant, $\lambda$ is the reorganization energy, and $\Delta G^{0}$ is the total Gibbs energy change for the reaction. By defining a Gibbs energy of activation,

$\Delta G^{*}=\frac{\left(\lambda+\Delta G^{0}\right)^{2}}{4 \lambda}$

we can put Eq. (1) into the standard Arrhenius form

$k_{\text {et }}=\frac{2 \pi}{\hbar} H_{\mathrm{DA}}^{2} \frac{1}{\sqrt{4 \pi \lambda k_{\mathrm{B}} T}} \exp \left(\frac{-\Delta G^{*}}{k_{\mathrm{B}} T}\right)$

For strongly exergonic electron transfer reactions that are activated by charge fluctuations in the environment [1,2], the activation energy is determined by the intersection point of the following thermodynamic potentials (Gibbs energies) of the reactants and products

$$
\begin{aligned}
& G_{\text {reactants }}=\frac{1}{2} Q_{1}^{2}\left(\frac{1}{4 \pi \varepsilon_{0}}\right)\left(\frac{1}{\varepsilon(0)}\right)\left(\frac{1}{a_{\mathrm{D}}}+\frac{1}{a_{\mathrm{A}}}-\frac{2}{d}\right) \\
& G_{\text {products }}=\frac{1}{2} Q_{2}^{2}\left(\frac{1}{4 \pi \varepsilon_{0}}\right)\left(\frac{1}{\varepsilon(\infty)+f_{1}[\varepsilon(0)-\varepsilon(\infty)]}\right)\left(\frac{1}{a_{\mathrm{D}}}+\frac{1}{a_{\mathrm{A}}}-\frac{2}{d}\right)
\end{aligned}
$$

The various terms are defined as follows. $G_{\text {reactants }}$ and $G_{\text {products }}$ are the total Gibbs energies of the reactants and products (including their ionic atmospheres). $Q_{1}$ and $Q_{2}$ are the charge fluctuations that build up on them. $\varepsilon(0)$ is the relative permittivity of the environment in the low frequency limit (static dielectric constant), $\varepsilon(\infty)$ is the relative permittivity of the environment in the high frequency limit $(\approx 2), a_{\mathrm{A}}$ is the radius of the acceptor in the transition state (including its ionic atmosphere), $a_{\mathrm{D}}$ is the radius of the electron donor in the transition state (including its ionic atmosphere), and $f_{1}$ is a constant $\left(0<f_{1}<1\right)$ that quantifies the extent of polar screening by the environment. The term $d$ is the distance between the electron donor and acceptor. A key prediction of Eqs (1-5) is that water is able to lower the activation energy of strongly exergonic electron transfer processes, and hence catalyse them (Fig.1).

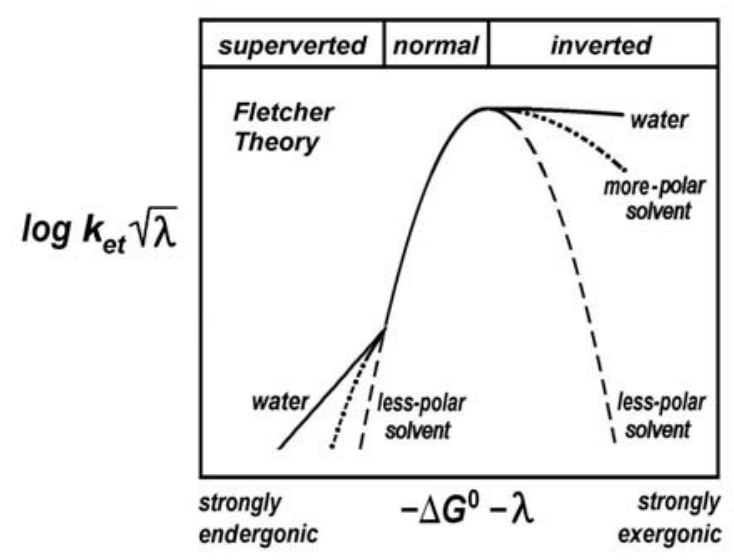


Fig. 1. The rate constant for electron transfer $\left(k_{\mathrm{et}}\right)$ as a function of the driving force $\left(-\Delta G^{0}\right)$ and reorganization energy $(\lambda)$ on the Fletcher theory [1, 2]. Note the powerful catalytic effect of polar solvents (such as water) on strongly exergonic reactions.

Given the catalytic effect of polar solvents on strongly exergonic electron transfer processes, it follows that if one wants to minimize damage from such reactions inside biological membranes then one must rigorously exclude water, which has a high relative permittivity $(78)$ at $25^{\circ} \mathrm{C}$. In the present work we point out that nature has contrived precisely this situation inside the photosynthetic reaction centre of plants, cyanobacteria and anoxygenic bacteria. Indeed, to inhibit photo-excited electrons from chemically reacting with nearby cofactors, scaffold proteins and membrane molecules, nature has evolved a highly non-polar, non-reducible environment inside the reductive region of photosynthetic membranes. This low-permittivity environment achieves its task by greatly increasing the electrostatic work required for electron donor and acceptor states to equalize their energies.

If nearby states readily attained the correct energies to exchange electrons, they would divert the electrons from their tunnelling pathways, and - catastrophically - trigger irreversible and degenerative chemical reactions. From these telling arguments we may therefore draw the following important conclusion: that a non-polar environment is needed to maintain the high efficiency and chemical integrity of the solar energy harvesting system, and that Darwinian evolution has converged on precisely this arrangement in all known photosynthetic species. We further remark that such a conclusion cannot be reached by conventional Marcus theory because it predicts (erroneously) that polar environments slow down the rates of highly exergonic electron transfer reactions. In fact, the very opposite is true. Accordingly, we must reluctantly conclude that Marcus theory fails to explain electron transfer in photosynthesis [15].

Since, in our view, the photosynthetic reaction centre has evolved to make all charge fluctuations energetically unfavourable (by raising $\lambda$ in Eq.1), how then are the biologically necessary electron transfer events selectively maintained at high rates? The answer is, by engineering high values of $H_{\text {DA }}$. This simply requires the placement of the relevant donor and acceptor states within $1.4 \mathrm{~nm}$ of each other, so that orbital overlap is well developed, and the tunnelling probability is high. (This is, we think, the generic reason why electron transfer in biological systems takes place through electron transport chains surrounded by hydrophobic amino acid residues.)

Finally, we remark that it is also desirable to exclude $\mathrm{O}_{2}$ (oxygen) from the vicinity of the trapping states in photosynthesis [16]. This is because oxygen is readily reducible to the free radical $\mathrm{O}_{2}{ }^{--}$ion (superoxide), which contains an unpaired electron. Although superoxide is not particularly reactive in itself, it is the precursor of a number of other oxygen-containing species that are (such as hydroxyl radicals), and these latter are known to attack double bonds or take part in hydrogen abstraction reactions [17]. 


\section{The General Structure of the Photosynthetic Reaction Centre}

The locus of oxygenic photosynthesis is the thylakoid. All oxygen-evolving photosynthetic organisms contain these flattened vesicles, which provide a highly structured environment in which the photosynthetic reactions take place. In plants, the thylakoid vesicles are generally housed inside larger structures called chloroplasts, which have an additional outer membrane. The solution internal to the thylakoid is referred to as the lumen, whereas the solution external to the thylakoid is referred to as the stroma. Often, to maximize the probability of light capture, thylakoids are stacked like piles of coins in structures known as grana.

Chemically, the thylakoid bilayer membranes of higher plants are composed primarily of galactolipids, which are electrically neutral (i.e. they do not have charged headgroups or tail-groups). Natural galactolipids are also lacking in low-lying acceptor states (i.e. they do not have conjugated double bonds). As a result of this unusual combination of features, the interior of a galactolipid membrane is electrically "quiet" (capable of only small charge fluctuations because of its low dielectric constant), nonpolar (hydrophobic), and resistant to electrochemical reduction. Astonishingly, galactolipids have also evolved independently in vertebrates as components of the myelin sheath of nerves, presumably for the same electrical reasons. The structural formula of a typical chloroplast galactolipid is shown in Fig.2.

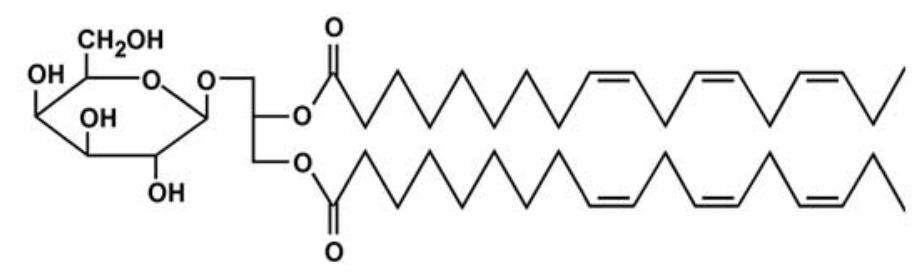

Fig. 2. Structural formula of a typical monogalactosyl diacyl glycerol (MGDG) that is found in thylakoid membranes. The rigorous name of the illustrated molecule is 1,2-di-(9,12,15)-octadecatrienoyl-3-O- $\beta$-D-galactopyranosyl-snglycerol, a widely distributed component of thylakoid membranes. The lipid tails, which we emphasize are not conjugated, are derived from alpha-linolenic acid (9,12,15-octadecatrienoic acid).

There are four major photosynthetic complexes inside a typical thylakoid membrane: photosystem I, photosystem II, cytochrome b6f, and ATP synthase. Due to the large distance between PSII and the cytochrome b6f complex, various hydrophobic quinones have evolved to shuttle electrons between them without leaving the membrane. In higher plants and cyanobacteria ("blue-green algae") the quinone is usually a derivative of 2,3-dimethyl-1,4-benzoquinone having an $n$-isoprenoid ( $n$ prenyl) side chain (plastoquinone, see Fig.3). In anoxygenic bacteria such as purple bacteria the corresponding quinone is usually a ubiquinone (Fig.4). In all species, the key step is the transformation of the quinone moiety into the corresponding hydroquinone moiety by two separate electron transfer reactions. 


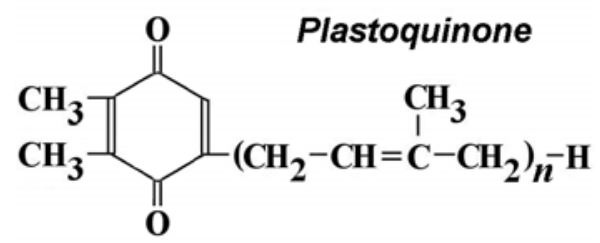

Fig. 3. Structural formula of a typical plastoquinone. The rigorous name of the illustrated molecule is 2,3-dimethyl-6-(n-prenyl)-1,4-benzoquinone. In most plants $n=9$.<smiles>CC=C(C)C=CC1=C(C)C(=O)C(C)=C(OC)C1=O</smiles>

Fig. 4. Structural formula of a typical ubiquinone. The rigorous name of the illustrated molecule is 2,3-dimethoxy-5-methyl-6-(n-prenyl)-1,4-benzoquinone. In most anoxygenic bacteria $n$ is 8,9 or 10 .

Anoxygenic bacteria contain only one major photosystem (known as the bacterial reaction centre, bRC) but this has a number of amino acid sequences that are very similar to those in photosystem II in both plants and cyanobacteria. Indeed, it seems likely that all these photosynthetic systems evolved inside a common ancestor. One curious difference between anoxygenic bacteria and plants, however, is that their photosynthetic apparatus is located inside a cytoplasmic membrane rather than a thylakoid membrane. This suggests that the thylakoid membrane may have evolved to keep oxygen away from the reaction centre. Despite this difference in membrane chemistry, the result of exposure to light is the same in both cases; a difference of electrochemical potential $\Delta \mu$ appears across the membrane. Famously, ATP synthase exploits this electrochemical potential difference to drive the phosphorylation of ADP [18].

From a structural point of view, the best understood of the anoxygenic photosynthetic reaction centres are those from bacteria in the family Rhodobacteraceae [19,20]. The redox cofactor organization in the bacterial reaction centre of Rhodobacter sphaeroides (formerly Rhodopseudomonas sphaeroides) is shown in Figure 5, [21]. A detailed picture of the mechanism of electron transfer inside the bacterial reaction centre has been built up from an epic series of experimental studies, summarized in refs [22-26]. 


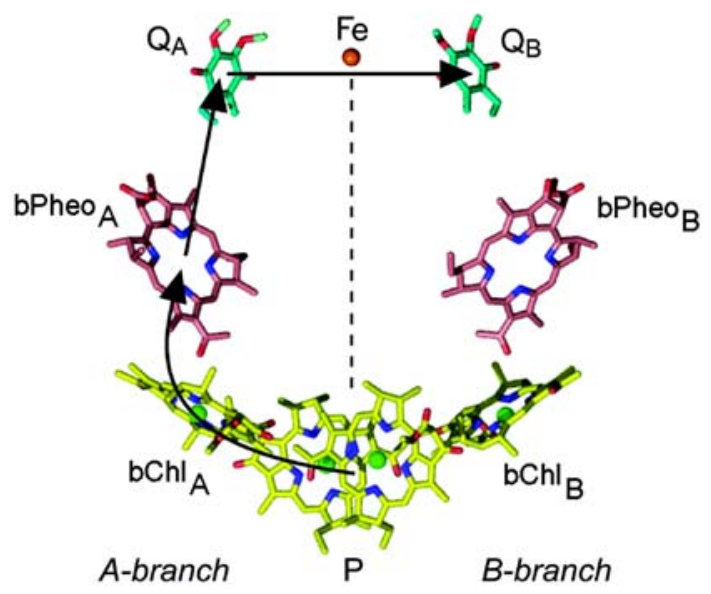

Fig 5. Spatial organization of electron trapping states ("redox cofactors") necessary for the normal functioning of the bacterial reaction centre of the nonoxygenic purple bacterium Rhodobacter sphaeroides. Neighbouring states are arranged within direct electron tunnelling distance of each other $(<1.4 \mathrm{~nm})$. Electron transport occurs principally through the A-branch (also known as the L-branch), beginning with the pair of bacteriochlorophyll (bChl) molecules, and ending with the quinone $Q B$. The redox cofactors are kept in position by stereoselective bonding with the helices of the $A$ and $B$ polypeptides. Image derived from ref. [21].

The primary electron donor is a pair of bacteriochlorophyll molecules located close to the lower face of the membrane. Photoexcitation of these molecules forms a charge separated singlet state. This, in turn, triggers electron transfer to the primary quinone molecule QA, via a monomeric bacteriochlorophyll (bChl) and a bacteriopheophytin (bPheo). Eventually, the photogenerated electron is passed to a secondary quinone molecule QB.

It can be seen from Fig. 5 that the redox cofactors are disposed around a two-fold axis of pseudo-symmetry. The arrows indicate the pathway of light-driven electron transfer. Despite its near-symmetric form, the reaction centre behaves asymmetrically - only one of its two branches (the A-branch) actually permits high-throughput electron tunnelling [27]. The same asymmetry is found in cyanobacteria and higher plants [28]. The mechanism by which electron tunnelling is inhibited in the B-branch has not yet been discovered.

\section{The Structure of Photosystem II}

We now turn our attention to photosystem II, which occurs in cyanobacteria and plants. Photosystem II is exceptional because it is the only biological machinery known that is able to oxidize water and generate molecular oxygen. In a recent series of papers, the architecture of photosystem II has been resolved at a resolution below $4.0 \AA$ [29-31]. First, Zouni et al. elucidated the crystal structure of photosystem II 
from Synechococcus elongatus at $3.8 \AA$ resolution [29], then Kamiya et al. reported the crystal structure of photosystem II from Thermosynechococcus vulcanus at $3.7 \AA$ resolution [30]. Finally, Ferreira et al. determined the architecture of photosystem II in the cyanobacterium Thermosynechococcus elongatus at $3.5 \AA$ resolution [31]. Ferreira et al. assigned all of the subunits of their photosystem II complex to specific genes and also provided a description of the protein environment of the various redox-active cofactors. In total, they assigned 3916 residues and successfully modelled the side chains. Their work revealed the three-dimensional structure of photosystem II at very high resolution.

It turns out that photosystem II consists of about 20 different protein subunits and 14 integrally bound lipids. But it contains only six redox cofactors that are able to trap electrons (or holes) in minima of Gibbs energy. These cofactors are the oxygenevolving complex (OEC), the amino acid residue tyrosine (Tyr), the reaction centre chlorophyll (Chl), pheophytin (Pheo), and the plastoquinone molecules, QA and QB $[32,33]$. All these cofactors except QB are bonded to a twisted pair of hydrophobic proteins known as D1 and D2. The D1 and D2 proteins form the scaffolding of the photosystem II complex. Each protein comprises five transmembrane helices (A to E) organized in a manner almost identical to that of the $\mathrm{L}$ and $\mathrm{M}$ subunits of the reaction centre of photosynthetic bacteria $[34,35]$. The plastoquinone QB is exceptional in that it may either diffuse inside the membrane, or bind to the reaction centre inside a special pocket. Pheo is a chlorophyll molecule lacking a central $\mathrm{Mg}^{2+}$ ion. The precise spatial organization of the redox cofactors in photosystem II of Thermosynechococcus elongatus is shown in Fig. 6.

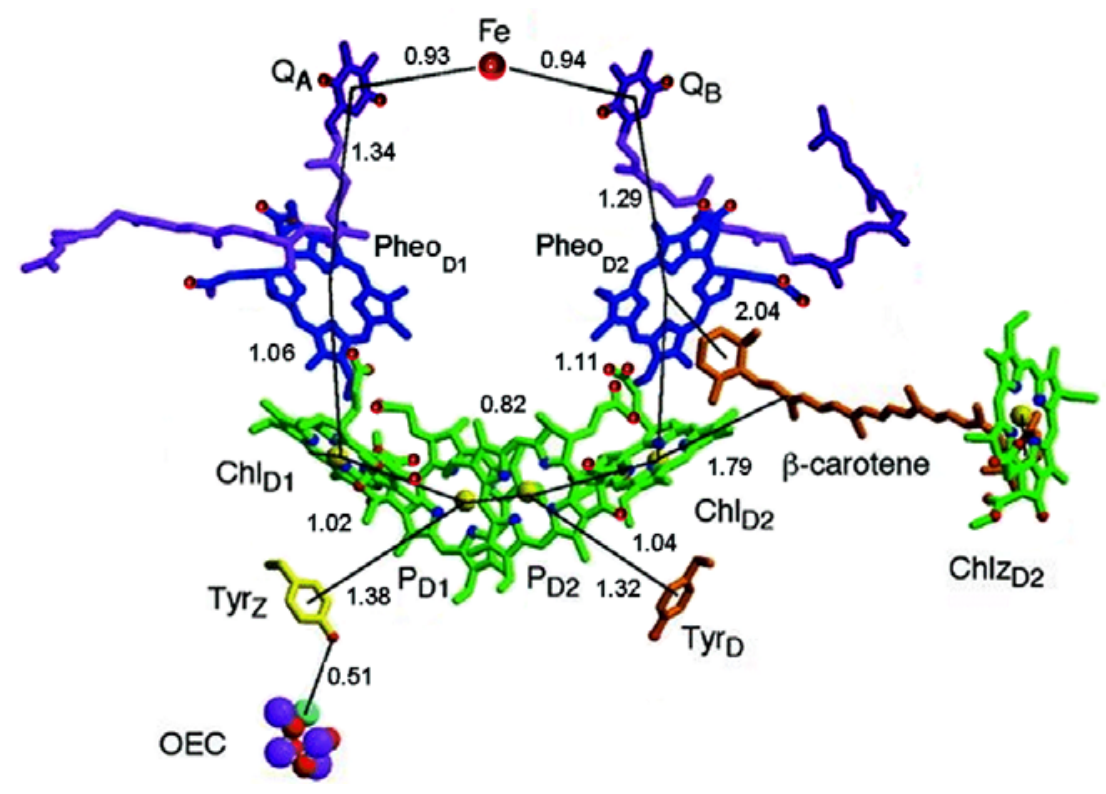

Fig. 6. Spatial organization of redox cofactors in photosystem II of the oxygenic cyanobacterium Thermosynechococcus elongatus, along the internal pseudotwofold axis. Distances in nanometers. As in the bacterial reaction centre shown in Fig. 5, all the neighbouring cofactors are arranged within electron tunnelling distance of each other $(<1.4 \mathrm{~nm})$. From Ferreira et al, Science $\underline{303}, 1831-1838$ (2004). Reprinted with permission from AAAS. 
The Chl* excited state is delocalized over a number of chlorophyll molecules, one of which is the Chl(D1) that is involved in the initial primary charge separation. The latter molecule injects an electron into Pheo (D1). Unlike accessory BChls in the bacterial reaction centre, which are anchored by histidines, there appears to be no obvious amino acid residue to anchor Chl (D1). At the other end of the electron pathway, the full reduction of $\mathrm{QB}$ requires two electrons and two protons, ultimately creating the plastohydroquinone $\mathrm{QBH}_{2}$, which diffuses into the membrane interior as a charge-neutral species. Meanwhile, a re-oxidized QB molecule diffuses back to the binding pocket and the process starts all over again. We can be reasonably sure that the binding pocket is connected with the polar aqueous phase (the stroma) because all the protons are sourced from there.

In our analysis which follows, the electron trapping states on the reducing side of PSII are denoted $T_{0}, T_{1}, T_{2}, T_{3}$, and $T_{4}$. These correspond to the redox cofactors P680, Ch1*, $\mathrm{Pheo}^{-}, \mathrm{QA}^{-}$and $\mathrm{QB}^{-}$, respectively. Many authors have shown that electrons tunnel spontaneously between these trapping states under the influence of an electrochemical potential $\Delta \mu$, which is generated by the absorption of light. What we now want to understand is the mechanism by which these tunnelling events occur, and how their rates are regulated.

\section{The Equivalent Circuit of the Electron Transport Chain in Photosystem II}

An equivalent circuit that captures the principal features of the electron transport chain in PSII is proposed in Fig. 7. At constant temperature and pressure, electrons tend to flow spontaneously from states of higher Gibbs energy to states of lower Gibbs energy, (i.e. from states of negative redox potential to states of positive redox potential). They do this provided only that (i) the lower states (electron acceptor states) are within tunnelling distance of the higher states (electron donor states), (ii) thermal fluctuations are available to overcome any activation barriers between the states, and (iii) energetic photons do not cause the electrons to move backwards against the gradient of Gibbs potential. Two feedback modes are here included, from $T_{1}$ to $T_{0}$ (radiative decay), and from $T_{2}$ to $T_{0}$ (non-radiative decay). The existence of further feedback modes is an open question.

Above a certain threshold light level, PSII operates as a delivery-on-demand system. That is to say, the electron trapping state $T_{4}$ is filled as often as required to replenish the quinone pool. The excess electrons that make this rapid-replenishment possible must be continually drained away by an "overflow" system. This may occur radiatively, via the red fluorescence, or non-radiatively, by a dark electron transfer process, or possibly by a third mechanism as yet unknown. 


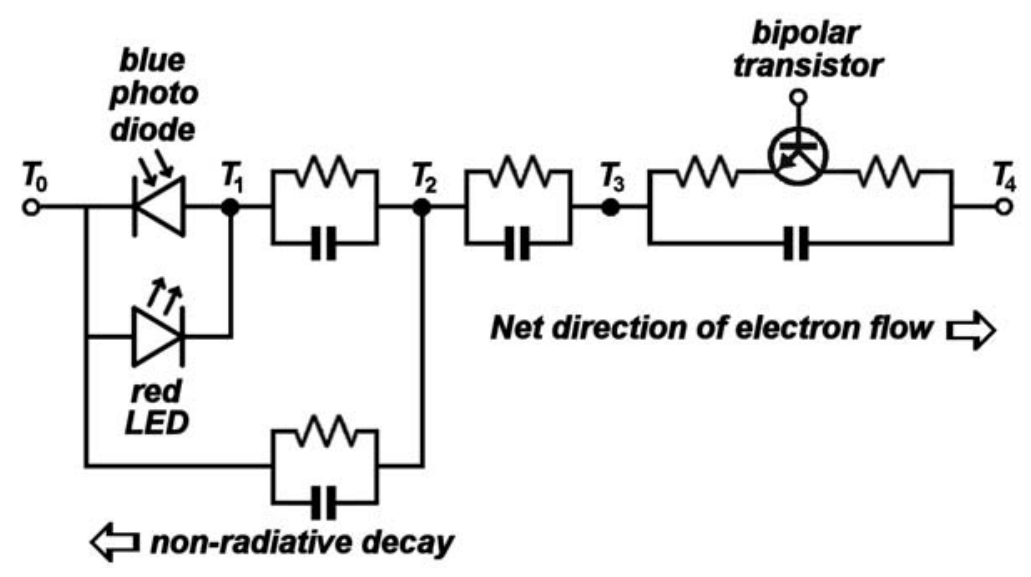

Fig. 7. Proposed equivalent circuit of the electron transport chain on the acceptor side (reducing side) of photosystem II. The electron trapping states are denoted $T_{0}, T_{1}, T_{2}, T_{3}$, and $T_{4}$. These correspond to the redox cofactors P680, $\mathrm{Chl}^{*}$, $\mathrm{Pheo}^{-}, \mathrm{QA}^{-}$and $\mathrm{QB}^{-}$. The non-linear circuit elements (diodes and transistor) correspond to self-regulatory features of PSII. The non-radiative decay of $T_{2}$ acts as an "overflow drain" when the pathway to $T_{4}$ is full or blocked.

In Fig.7, the blue photodiode represents the effect of illumination on PSII. No current flows in the dark; but current flows in the light. The first stable product is a vibrationally relaxed but electronically excited state of chlorophyll $\left(T_{1}\right)$. The red LED represents the well-known energy loss from this state by fluorescence at $680 \mathrm{~nm}$. To minimize this energy loss, electron tunnelling between $T_{1}$ and $T_{2}$ takes place very quickly indeed, with a time constant below $20 \mathrm{ps,} \mathrm{yielding} \mathrm{Chl}^{+}$and $\mathrm{Pheo}^{-}$. This latter species constitutes the trapping state $T_{2}$. The reversible potentials and lifetimes of all the known trapping states in PSII are summarized in Table 1.

\begin{tabular}{|c|c|c|c|c|}
\hline PSII & $\begin{array}{l}E_{\mathrm{R}}(\mathrm{mV}) \\
\text { (observed) }\end{array}$ & $\begin{array}{l}E_{\mathrm{R}}(\mathrm{mV}) \\
(\mathrm{Eq} .6)\end{array}$ & $\begin{array}{l}\text { Lifetime } \\
\text { (observed) }\end{array}$ & $\begin{array}{l}\text { Lifetime } \\
\text { (Eq. 6) }\end{array}$ \\
\hline$T_{1} \quad \mathrm{Chl}$ & $-620 \pm 100$ & -621 & $<20 \mathrm{ps}$ & $2 \mathrm{ps}$ \\
\hline$T_{2} \quad$ Pheo & $-499 \pm 100$ & -503 & $100-450 \mathrm{ps}$ & $200 \mathrm{ps}$ \\
\hline$T_{3} \quad \mathrm{QA}$ & $-150 \pm 100$ & -149 & $100-600 \mu \mathrm{s}$ & $200 \mu \mathrm{s}$ \\
\hline$T_{4} \quad \mathrm{QB}$ & $+50 \pm 100$ & +88 & $1 \mathrm{~ms}$ to $100 \mathrm{~s}$ & $2 s$ \\
\hline
\end{tabular}

Table 1. Approximate reversible potentials (native redox potentials measured with respect to the standard hydrogen electrode potential) and lifetimes of the trapping states in the electron transport chain of photosystem II. Data derived from refs [36-41].

The next act of electron transfer, between $\mathrm{Pheo}^{-}$and the membrane-bound plastoquinone QA, is also surprisingly fast, having a time constant of 100-450 ps. The final act of electron transfer, between the membrane-bound plastoquinone $\mathrm{QA}^{-}$and the free plastoquinone $\mathrm{QB}$, is considerably slower, and may be delayed still further by 
the time taken for free plastoquinone molecules QB to diffuse to the binding pocket, or to undergo some other thermally activated process. Its time constant is not known with certainty, but is probably in the range $(100-600 \mu \mathrm{s})$. The QA/QB electron transfer process is mediated by a non-heme iron (II) atom, an arrangement that functions as a bipolar transistor, but this process is so complex that a more extensive discussion is deferred to a separate paper. The reversible potential of the QA/QA couple is more negative (less stable) than that of the $\mathrm{QB} / \mathrm{QB}^{-}$couple, doubtless because the latter is stabilized by a hydrogen-bonded (more polar) environment. Finally, to satisfy Kirchhoff's Current Law, the overall system must ultimately form a complete electrical circuit (not shown in the figure). It does this by driving protons through the membrane-spanning ATP synthase system [18].

Fig. 8 is a semi-logarithmic plot of the lifetimes $(\tau)$ of various trapped species in PSII as a function of their reversible potentials $E_{\mathrm{R}}$ (volts versus S.H.E.). The straight line is drawn according to the relation

$$
\left(E_{\mathrm{R}} / \mathrm{V}\right)=0.070+0.0591 \log (\tau / \mathrm{s})
$$

Due to the wide range of energies and timescales involved [36-41], the graph is robust against large measurement errors $( \pm 100 \mathrm{mV}$ in potential, \pm an order-of-magnitude in lifetime), so we can be confident of the general trend. It is clear that the high energy states are the most short-lived. Further, the backward rates (on average) are about 10,000 times slower than forward rates. The electric field strength is also extraordinary - the system drops $600 \mathrm{mV}$ in about $4 \mathrm{~nm}$, implying a field strength of $\sim 150 \mathrm{MV} / \mathrm{m}[42,43]$. This is close to the dielectric breakdown strength of most cell membranes. In other words, if the field strength were slightly higher, sparks would be generated!

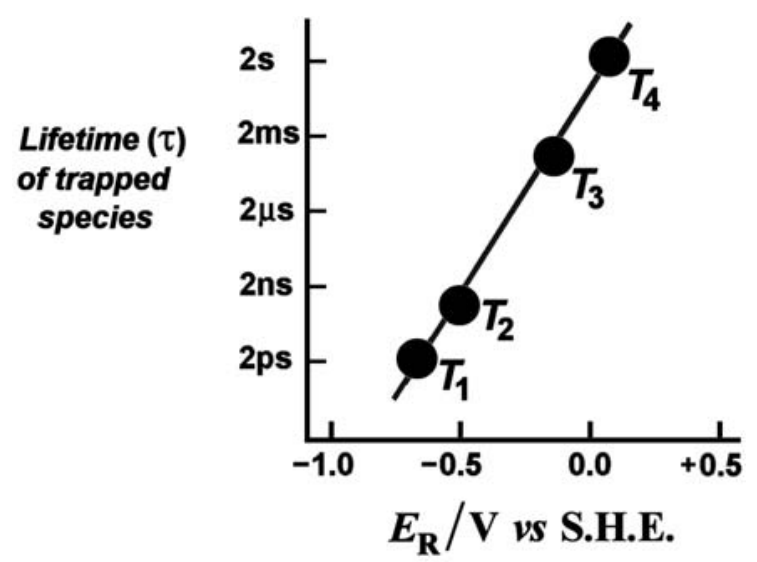

Fig. 8. The lifetimes of the trapping states in the electron transport chain of PSII, as a function of their reversible potentials. Data compiled from refs [36-41].

\section{Gibbs Energy Profiles Inside Photosystem II}

To gain a deeper insight into the operation of photosystem II we need to construct the Gibbs energy profiles. Some general bounds on the placement of these are provided 
by the laws of thermodynamics. For example, on the standard hydrogen scale, the oxygen evolution reaction

$2 \mathrm{H}_{2} \mathrm{O} \leftrightarrows \mathrm{O}_{2}+4 \mathrm{H}^{+}+4 \mathrm{e}^{-}$

has a thermodynamic equilibrium potential of

$E_{\text {eq }}=1.228-0.0591 \mathrm{pH}+0.0147 \log p\left(\mathrm{O}_{2}\right)$

at $25^{\circ} \mathrm{C}$. Thus at $\mathrm{pH} 7.0$ and $25^{\circ} \mathrm{C}$, we find that under standard conditions the oxidation of water (evolution of oxygen) requires $E>+0.814 \mathrm{~V}$. In other words, we can be sure that the Gibbs energy minimum of the Chl/Pheo/QA complex occurs at $E$ $>+0.814 \mathrm{~V}$, because oxygen would not be able to evolve otherwise. Here, we place the minimum at $+1.114 \mathrm{~V}$, since a $300 \mathrm{mV}$ overpotential is comparable with the overpotential of the best metal oxide catalysts for oxygen evolution. Similarly, we can be sure that the Gibbs energy minimum of vibrationally-relaxed Ch1*/Pheo/QA occurs at least $1.82 \mathrm{~V}$ above the Gibbs energy minimum of Chl/Pheo/QA, because the known fluorescence emission of PSII occurs at $680 \mathrm{~nm}$ (which corresponds to $1.82 \mathrm{eV}$ ). Furthermore, the activation energy for the charge separation step

$\mathrm{Chl} * / \mathrm{Pheo} / \mathrm{QA} \rightarrow \mathrm{Chl}^{+} / \mathrm{Pheo}^{-} / \mathrm{QA}$

must be very small, because that particular reaction occurs with remarkable speed, being essentially complete within 20 ps. Gibbs energy profiles that satisfy these many and various constraints are shown in Fig. 9.

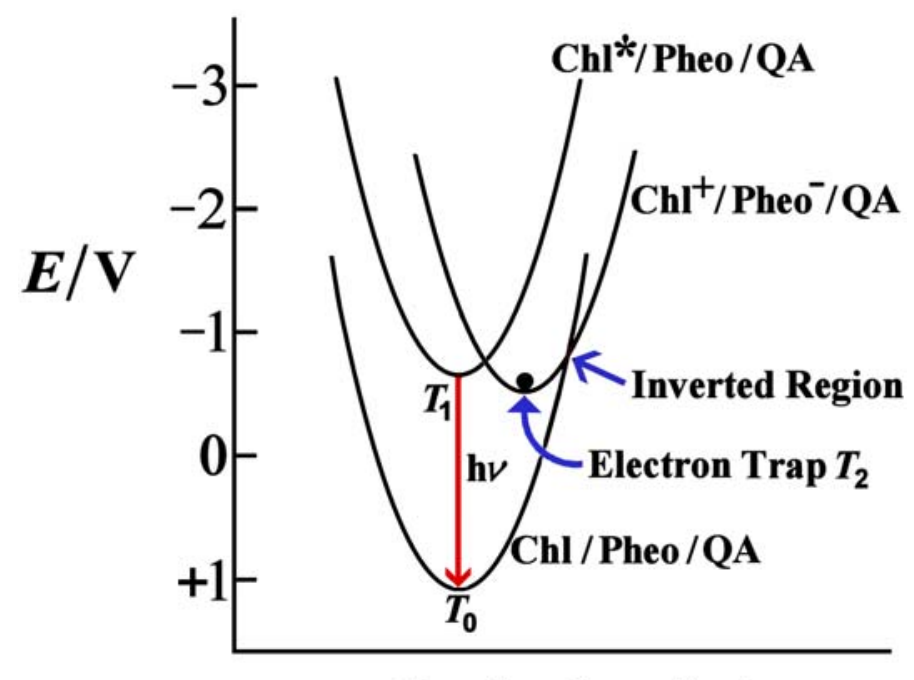

Reaction Co-ordinate

Fig. 9. Superimposed Gibbs energy profiles for different states of the Chl/Pheo/QA complex that arise inside the electron transport chain of PSII during photo-induced charge separation. The initial state is denoted $\mathrm{Chl} / \mathrm{Pheo} / \mathrm{QA}$, the electronically excited state is denoted $\mathrm{Ch} / \mathrm{Pheo} / \mathrm{QA}$, and the charge-separated state is denoted $\mathrm{Chl}^{+} / \mathrm{Pheo}^{-} / \mathrm{QA}$. An electron is shown in the trap $T_{2}$. The downward arrow indicates the red fluorescence emission. For clarity, we have omitted the Gibbs energy profiles of the trapping state $T_{3}$ and any triplet states. 
Given the known thermodynamic and kinetic constraints on PSII there is surprisingly little leeway in the placement of the parabolas in Fig. 9. The beauty of this diagram is that the "central mystery" of photosynthesis — that is, the reason why the electron in the high-energy $T_{2}$ state does not instantaneously decay back to the low-energy ground state $T_{0}$ - is now revealed. We see that there are, in fact, two activation energy barriers that inhibit this process, with the height of both barriers determined by the width of the Gibbs energy parabola of $\mathrm{Chl}^{+} / \mathrm{Pheo}^{-} / \mathrm{QA}$. The situation is shown in close-up in Fig.10. Evidently, the narrower the Gibbs energy profile, the larger the activation energies of the escape routes, and the longer the lifetime of the chargeseparated state $T_{2}$.

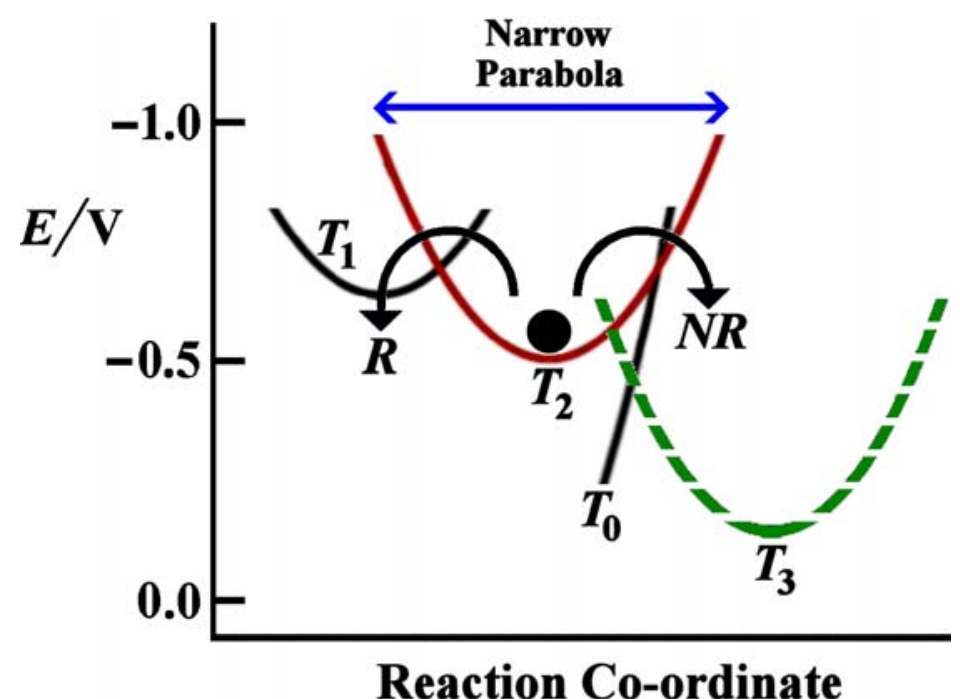

Fig. 10. Superimposed Gibbs energy profiles in the vicinity of the electron trap $T_{2}$. Trapping is thermodynamically reversible, so the electron can return to $T_{0}$ radiatively (R) via $T_{1}$ or non-radiatively (NR) via the inverted region. Both routes are kinetically hindered by the extreme narrowness of the Gibbs energy parabola, however. This narrowness is conferred by the extremely non-polar environment surrounding $T_{2}$. Trapping state $T_{3}$ is the biological goal.

Based on recent advances in the theory of electron transfer [1, 2], we can now understand how nature manages to stabilize the charge-separated state $T_{2}$. An extremely narrow Gibbs energy parabola is formed by packing non-polar amino acid residues around it. This makes it extremely difficult for charge fluctuations to build up. Conversely, if a polar species such as water happened to encroach upon $T_{2}$, the Gibbs energy parabola would broaden in the inverted region [2], non-radiative decay would be strongly catalyzed, and photosynthesis would switch off. A single molecule of water might be sufficient to achieve this. Given this sensitivity to environmental polarity, it is interesting to ask if all the other trapping states in the conducting branch of PSII are surrounded by non-polar amino acid residues in a similar way. Strong evidence that this is indeed the case (based on recent $\mathrm{x}$-ray data) is now presented. 


\section{The Number and Polarity of Amino Acid Residues That Surround the Electron Trapping States in Photosystem II}

According to recent studies of cofactor arrangements in the electron transport chain of photosystem II, the amino acid residues are arranged in the following way [29, 44].

Chl (D1) has four extremely hydrophobic phenylalanine residues in its neighbourhood (D1 Phe119, D1 Phe158, D1 Phe180 and D1 Phe182). It is also possibly hydrogen bonded via its 13(1) keto group to D2 His197 via a water molecule (although this has not yet been resolved by x-ray crystallography).

Pheo (D1) is hydrogen bonded via its 13(1) keto group to D1 Gln130 and to its ester carbonyl groups by the hydrophobic residues D1 Tyr126 and D1 Tyr147, and is also close to D2 Phe257.

The immobile quinone QA is surrounded by the hydrophobic residues D2 Ile213, D2 Met246, D2 Ala249 and D2 Ala260, and is also sandwiched between the extremely hydrophobic residues D2 Trp253 and D2 Leu267. There are also two hydrogen bonds to the oxygen atoms of the quinone headgroup, one from D2 His214 (which also ligates a non-heme iron) and one from the backbone nitrogen of D2 Phe261.

The non-heme iron (which is not a trapping state for the electron) is surrounded by D1 His215, D1 His272, D2 His214 and D2 His268. There is also a bicarbonate ion that acts as a bidentate ligand.

Finally, the mobile quinone QB resides in a binding pocket composed of yet more hydrophobic residues, including D1 Met214, D1 Leu218, D1 Ala251, D1 Phe255, D1 Phe265 and D1 Leu271. However, QB is also hydrogen bonded to D1 His215 and the side chain oxygen of D1 Ser264.

A list of amino acid residues, sorted according to the relative permittivity of their terminal functional groups, is presented in Table 2 . The hydrophobicity index $H$ is a measure of how insoluble the parent amino acid is in water. Here, we have assumed a linear correlation between the hydrophobicity of the free amino acid and the hydrophobicity of the bound amino acid. The $H$ values in the table are normalized so that the most hydrophobic residue is given a value of 100 relative to glycine, which is arbitrarily assigned a value 0 . The values below glycine are obtained by extrapolation.

Note that the highly polar carboxylic acid functional groups in each amino acid do not interfere with the electron tunnelling pathway because they are "locked up" inside peptide linkages. Phenylalanine, tryptophan and tyrosine have large aromatic ring terminations, which explains why they have very low relative permittivity (high hydrophobicity) when bonded inside proteins. They "look like benzene". Amino acids with alkyl terminations (isoleucine, leucine, valine, and alanine) also provide strong hydrophobicity inside proteins. Methionine uniquely has a chemically inert thiol ether side chain. Apart from this select group, all other amino acids have high permittivity terminations.

Among the hydrophilic amino acids, three (tyrosine, threonine, and serine) have hydroxyl terminations and readily form hydrogen bonds with water. A further five have polar terminations containing lone pairs of electrons (cysteine, glycine, 
glutamine, histidine, and proline), and these are not only hydrophilic but may also act as ligands for metal ions. Finally, four other amino acids have end groups that are electrically charged at $\mathrm{pH} 7$ (arginine, lysine, glutamic acid, and aspartic acid). Overall, it is clear that nature has a limited choice of amino acids for constructing pathways of low relative permittivity. Given this limited choice, it is interesting to ask which particular amino acid residues actually surround the electron trapping states in photosystem II. The results are collected in the final column of Table 2. Clearly, the majority of amino acid residues along the electron tunnelling pathway are hydrophobic, i.e. have sufficiently low permittivity to suppress unwanted side reactions. Indeed, the amino acid residues are predominantly those having alkyl or aromatic side chains, whilst those with ionic (acidic or basic) side chains are absent. These data are fully consistent with the new theory of electron transfer [1,2].

\begin{tabular}{|l|c|c|c|}
\hline \multicolumn{1}{|c|}{ Name } & & $\boldsymbol{H}$ & Location \\
\hline & & & \\
\hline Phenylalanine & Phe & 100 & $\begin{array}{c}\text { D1 Phe119, D1 Phe158, D1 Phe180, } \\
\text { D182, D1 Phe255, D2 Phe257, } \\
\text { D2 Phe261, D1 Phe265 }\end{array}$ \\
\hline Isoleucine & Ile & 99 & D2 Ile213 \\
\hline Leucine & Leu & 97 & D1 Leu218, D2 Leu267, D1 Leu271 \\
\hline Tryptophan & Trp & 97 & D2 Trp253 \\
\hline Valine & Val & 76 & \\
\hline Methionine & Met & 74 & D1 Met214, D2 Met246 \\
\hline Tyrosine & Tyr & 63 & D1 Tyr126, D1 Tyr147 \\
\hline Alanine & Ala & 41 & D2 Ala249, D1 Ala251, D2 Ala260 \\
\hline & & & \\
\hline Cysteine & Cys & 49 & \\
\hline Threonine & Thr & 13 & \\
\hline Histidine & His & 8 & D1 His215, D2 His214 \\
\hline Glycine & Gly & 0 & \\
\hline Serine & Ser & -5 & \\
\hline Glutamine & Gln & -10 & D1 Ser264 \\
\hline Proline & Pro & -46 & D1 Gln130 \\
\hline & & & \\
\hline Arginine (+) & Arg & -14 & \\
\hline Lysine (+) & Lys & -23 & \\
\hline Glutamic (-) & Glu & -31 & \\
\hline Aspartic (-) & Asp & -55 & \\
\hline
\end{tabular}

Table 2. The amino acid residues that surround the electron trapping states in photosystem II, sorted according to the relative permittivity of their terminal functional groups (lowest permittivity on top). The Hydrophobicity Index values (H) from ref [45]. Residue locations from Ref [29]. 
Finally, it may be noted that, for any redox couple inside the pathway, the non-polar environment destabilizes the more highly charged states, making them thermodynamically harder to form. Hence for positively charged redox couples, such as $\mathrm{Fe}(2+) / \mathrm{Fe}(3+)$, the non-polar environment causes the redox potential to shift in a positive direction. Conversely, for negatively charged redox couples, like QA/QA ${ }^{-}$, the non-polar environment causes the redox potential to shift in a negative direction. This explains, in part, how PSII is able to achieve its phenomenally high oxidizing and reducing potentials.

\section{Conclusions}

Photosystem II has evolved to capture solar energy efficiently, and then store that energy in chemical bonds. The first step involves the photochemical excitation of stationary electrons. The second step involves the lateral tunnelling of those electrons through an electron transport chain. In the present work, we have explored the electronic structure and mechanism by which this electron transport chain operates. We find that the experimental data are consistent with a sequence of non-adiabatic electron transfer processes triggered by charge fluctuations in the environment of each electron trapping species. We can find no evidence that electron transfer is triggered by dielectric fluctuations (Marcus-Hush theory).

By the principle of least action, electrons tend to tunnel through those regions of space that have the most positive electrostatic potential. Not surprisingly, therefore, PSII has evolved a well-defined pathway of positive electrostatic potential to direct electrons to where it wants them. In the present work, we have proposed an equivalent circuit that models many of the electrical and optical features of the electron transport chain (Fig. 7). Our equivalent circuit is fully consistent with the biochemistry, electrochemistry, thermodynamics, x-ray crystal structure, femtosecond spectroscopy, and quantum mechanics of PSII.

Based on recent developments in the theory of electron transfer (which, for the first time, include the role of charge fluctuations in the electron transfer process $[1,2])$, and based on literature data, we have also determined the Gibbs energy diagram for the primary act of charge separation in photosynthesis. The results are shown in Figs. 9 and 10 . We find that the electron resides briefly in the unstable state $T_{2}$, before moving on to the more stable state $T_{3}$. While in the unstable state $T_{2}$, it may lose energy radiatively after a "normal" electron transfer process, or it may lose energy non-radiatively via an "inverted" electron transfer process. The competitive nature of these processes is evident. However, both processes are suppressed by the extreme narrowness of the Gibbs energy parabola of the $T_{2}$ state. The narrowness of the Gibbs energy parabola is conferred by the highly non-polar amino acid residues that surround the $T_{2}$ state. The resulting meta-stability of the $T_{2}$ state allows sufficient time for the electron to transfer successfully to $T_{3}$. This elegant arrangement finally explains the high efficiency of the primary charge separation step in photosynthesis.

Fig. 10 also explains the well-known, but puzzling, experimental finding that the rate of primary charge separation in photosynthesis increases upon cooling to cryogenic temperatures. Martin and co-workers [46, 47] were the first to quantify this 
phenomenon, by measuring the rate of electron transfer in isolated reaction centres of Rhodobacter sphaeroides. In the late nineteen eighties they found a time constant of $\sim 2.8 \mathrm{ps}$ at room temperature falling to $\sim 1.2 \mathrm{ps}$ at $10 \mathrm{~K}$. Since then, the acceleration of the rate of primary charge separation by cooling has been widely confirmed [48]. Now we have the explanation. The activation energies of the backward steps are much higher than the activation energies of the forward steps. As a consequence, the rate constants of the forward reactions are only slightly decreased by temperature, whereas their populations are greatly increased. The overall effect is an increase in rate.

An unwanted side reaction in PSII is the reduction of trace oxygen to hydrogen peroxide:

$\mathrm{O}_{2}+\mathrm{e}^{-} \leftrightarrows \mathrm{O}_{2}^{\cdot-}$

$\mathrm{O}_{2}^{\cdot-}+\mathrm{H}^{+}+\mathrm{e}^{-} \leftrightarrows \mathrm{HO}_{2}^{-}$

$\mathrm{HO}_{2}^{-}+\mathrm{H}^{+} \leftrightarrows \mathrm{H}_{2} \mathrm{O}_{2}$

where $\mathrm{O}_{2}{ }^{--}$is the superoxide ion and $\mathrm{HO}_{2}{ }^{-}$is the hydroperoxide ion. The overall reaction

$\mathrm{O}_{2}+2 \mathrm{H}^{+}+2 \mathrm{e}^{-} \leftrightarrows \mathrm{H}_{2} \mathrm{O}_{2}$

has a thermodynamic equilibrium potential

$E_{\mathrm{eq}}=0.682-0.0591 \mathrm{pH}+0.0295 \log \frac{p\left(\mathrm{O}_{2}\right)}{\left[\mathrm{H}_{2} \mathrm{O}_{2}\right]}$

at $25^{\circ} \mathrm{C}$. This implies that, at $\mathrm{pH} 7.0$ and $25^{\circ} \mathrm{C}$, and assuming $p\left(\mathrm{O}_{2}\right)=10^{-6}$ and $\left[\mathrm{H}_{2} \mathrm{O}_{2}\right]$ $=10^{-6} \mathrm{M}$, the reduction of trace oxygen may potentially occur wherever $E<+0.327$ $\mathrm{V}$. The clear implication is that reactive oxygen species may form in any electron trapping state if oxygen is not excluded. It follows that oxygen and water must both be carefully regulated inside PSII if the system is to operate successfully. On the other hand, if oxygen and water enter the electron transfer pathway simultaneously, then the photosynthetic reaction centre will be subjected to powerful oxidative stress (destruction of the protein scaffold).

Regarding the overall functioning of PSII, a number of commercially available herbicides have been developed to disrupt it. One of these is DCMU (3-(3,4dichlorophenyl)-1,1-dimethylurea), which prevents the mobile quinone QB from docking into its binding pocket on the D1 protein. It also stimulates the chlorophyll fluorescence at $680 \mathrm{~nm}$, but why? The reason is now obvious from Fig. 7. When DCMU blocks electron flow, the occupancy of the $T_{1}$ state increases, and the red fluorescence is stimulated. Conversely, when DCMU is removed, the occupancy of the $T_{1}$ state decreases, and the red fluorescence is quenched. By similar reasoning, the addition of oxidized QB should enhance the electron flow, decrease the occupancy of the $T_{1}$ state, and therefore quench the chlorophyll fluorescence. This is exactly what is observed experimentally [49]. 
Finally, we conclude that the asymmetric conductance of the near-symmetric reaction centre (Fig. 5) has almost certainly evolved to prevent the highly reactive semiquinone intermediate $\mathrm{QA}^{--}$from physically escaping. Thus, natural selection has sacrificed the photochemical functioning of the D2 branch of photosystem II ( $B$ branch of the bacterial reaction centre) in order to construct a semiquinone trapping state at the point where the photogenerated electrons exit the reaction centre. This strategy undoubtedly has a strong survival advantage, because it prevents the proliferation of unwanted free radical reactions involving $\mathrm{QA}^{{ }^{-}}$. Originally, the nonheme $\mathrm{Fe}$ (II) atom may perhaps have allowed the stabilization of the unpaired electron over two QA molecules. But today it clearly functions as an "electron window" (superexchange conduit) between the trapped QA and the free QB.

\section{Acknowledgements}

I should like to thank Christian Amatore (École Normale Supérieure, Paris) for drawing the problem of charge transfer in PSII to my attention; and Frank Müh (Freie Universität, Berlin) and Athina Zouni (Technische Universität, Berlin) for their generous help in interpreting the X-ray crystal structures of PSII.

\section{References}

[1] Fletcher S (2007) J Solid State Electrochem 11:965

[2] Fletcher S (2008) J Solid State Electrochem 12:765

[3] Marcus RA (1956) J Chem Phys 24:966

[4] Marcus RA (1956) J Chem Phys 24:979

[5] Marcus RA (1963) J Chem Phys 38:1858

[6] Marcus RA (1964) Ann Rev Phys Chem 15:155

[7] Marcus RA (1965) J Phys Chem 43:679

[8] Marcus RA (1994) J Phys Chem 98:7170

[9] Marcus RA (1997) "Electron Transfer Reactions in Chemistry: Theory and Experiment” Malmström BG, Ed., Nobel Lectures, Chemistry 1991-1995. World Scientific Publishing, Singapore

[10] Hush NS (1961) "Proc. Fourth Moscow Conference of Electrochemistry (1956)" (English Translation 1961) Consultants Bureau, New York

[11] Hush NS (1958) J Chem Phys 28:962

[12] Hush NS (1961) Trans Faraday Soc 57:557 
[13] Hush NS (1999) J Electroanal Chem 470:170

[14] Dirac PAM (1930) "The Principles of Quantum Mechanics", Clarendon Press, Oxford.

[15] Marcus RA, Sutin N (1985) in: "Antennas and Reaction Centers of Photosynthetic Bacteria", Michel-Beyerle ME, Ed., Springer-Verlag, Berlin, W. Germany pp. 226-233

[16] Khorobrykh S, Mubarakshina M, Ivanov B (2004) Biochimica et Biophysica Acta 1657:164

[17] Afanas'ev IB (1991) "Superoxide Ion: Chemistry and Biological Implications" CRC Press, Boca Raton, Florida

[18] Mitchell P (1961) Nature 191:144

[19] Deisenhofer J, Norris JR (1993), Eds., "The Photosynthetic Reaction Centre" Vols. I and II, Academic Press, San Diego, California

[20] Blankenship RE, Madigan MT, Bauer CE, (1995), Eds., "Anoxygenic Photosynthetic Bacteria", Kluwer, Dordrecht, The Netherlands

[21] Wakeham MC, Jones MR (2005) Biochem Soc Trans 33:851

[22] Woodbury NW, Allen JP (1995) in "Anoxygenic Photosynthetic Bacteria", Blankenship RE, Madigan MT, Bauer CE, Eds., pp. 527-557, Kluwer, Dordrecht, The Netherlands

[23] Parson WW (1996) in "Protein Electron Transfer" Bendall DS, Ed., pp. 125160, BIOS Scientific Publishers Ltd, Oxford

[24] Hoff AJ, Deisenhofer J (1997) Physics Reports 287:1

[25] Okamura MY, Paddock ML, Graige WS, Feher G (2000) Biochim Biophys Acta 1458:148

[26] Wraight CA (2004) Front Biosci 9:309

[27] Vermeglio A, Clayton RK (1977) Biochim Biophys Acta 461:159

[28] Bouges-Bocquet B (1973) Biochim Biophys Acta 314:250

[29] Zouni A, Witt HT, Kern J, Fromme P, Krauss N, Saenger W, Orth P (2001) Nature 409:739

[30] Kamiya N, Shen J-R (2003) Proc Natl Acad Sci USA 100:98

[31] Ferreira KN, Iverson TM, Maghlaoui K, Barber J, Iwata S (2004) Science 303:1831

[32] Debus R (1992) Biochim Biophys Acta 1102:269 
[33] Klimov VV (2003) Photosynthesis Research 76:247

[34] Deisenhofer J, Epp O, Miki K, Huber R, Michel H (1985) Nature 318:618

[35] Allen JP, Feher G, Yeates TO, Komiya H, Rees DC (1988) Proc Natl Acad Sci USA 85:8487

[36] Orr L, Govindjee (2007) Photosynthesis Research 91:107

[37] Ishikita H, Biesiadka J, Loll B, Saenger W, Knapp E-W (2006) Angew Chem Int Ed 45:1964

[38] Ishikita H, Saenger W, Biesiadka J, Loll B, Knapp E-W (2006) Proc Natl Acad Sci USA 103:9855

[39] Johnson GN, Rutherford AW, Krieger A (1995) Biochimica et Biophysica Acta (Bioenergetics) 1229:202

[40] Klimov VV, Krasnovskii AA (1981) Photosynthetica 15:592

[41] Diner BA, Rappaport F (2002) Ann Rev Plant Biol 53:551

[42] Tanaka S, Marcus RA (1997) J Phys Chem B 101:5031

[43] Marcus RA, Sutin N (1985) Biochimica et Biophysica Acta (Reviews on Bioenergetics) 811:265

[44] Loll B, Kern J, Saenger W, Zouni A, Biesiadka J (2005) Nature 438,:1040

[45] Monera OD, Sereda TJ, Zhou NE, Kay CM, Hodges RS (1995) J Peptide Sci $1: 319$

[46] Martin J-L, Breton J, Hoff AJ, Migus A and Antonetti A (1986) Proc Natl Acad Sci USA 83:957

[47] Breton J, Martin J-L, Fleming GR, Lambry J-C (1988) Biochemistry 27:8276

[48] Aartsma TJ, Amesz J (1996) Photosynthesis Research 48:99

[49] Kurreck J, Schödel R, Renger G (2000) Photosynthesis Research 63:171 